\title{
In Vitro Activity of Tetracycline Analogs against Multidrug-resistant and Extensive drug resistance Clinical Isolates of Mycobacterium tuberculosis
}

\section{Qi Ouyang}

Shenzhen University

Dachuan Lin

Shenzhen University

Guofang Deng

The Third People's Hospital of Shenzhen

\section{Zhihua Wen}

Yuebei Second People's Hospital

Houming Liu

The Third People's Hospital of Shenzhen

Hongzhen Li

Yuebei Second People's Hospital

Yi Cai

Shenzhen University

Xinchun Chen ( $\nabla$ chenxinchun@szu.edu.cn )

Shenzhen University https://orcid.org/0000-0002-2101-9013

\section{Research}

Keywords: Tetracycline, Multiple-drug resistance, Doxycycline, Mycobacterium tuberculosis

Posted Date: November 27th, 2019

DOI: https://doi.org/10.21203/rs.2.17754/v1

License: (c) (i) This work is licensed under a Creative Commons Attribution 4.0 International License. Read Full License 


\section{Abstract}

Background Multidrug-resistant tuberculosis (MDR-TB) has become a big threaten to global health. The current strategy for treatment of MDR-TB and extensive drug resistant tuberculosis (XDR-TB) is with low efficacy and high side effect. While new drug is fundamental for cure MDR-TB, repurposing the Food and Drug Administration (FDA)-approved drugs represents an alternative soluation with less cost.

Methods The activity of 8 tetracycline-class antibiotics against mycobacterium tuberculosis (M.tb ) were determined by Minimum Inhibitory Concentration (MIC) in vitro. A transposon M.smeg libraries was generated by using the Harm phage and then used to isolate the conditional growth mutants in doxycycline containing plate. 11 mutants were isolated and genomic DNAs were extracted using the cetyltrimethyl ammonium bromide (CTAB) method and analyzed by whole genome sequencing.

Results We found that three of eight drugs efficiently inhibited mycobacteria growth under the peak plasma concentration in the human body. Further tests showed these three tetracycline analogs (demeclocycline, doxycycline and methacycline) had antimicrobial activity against seven clinical isolates, including MDR and XDR strains. Among them, Doxycycline had the lowest MICs in all mycobacteria strains tested in this study. By using a transposon library, we identify the insertion of transposon in two genes, porin and MshA, associate with the resistant to doxycycline.

Conclusions Our findings show that tetracycline analogs such as doxycycline, has bactericidal activity against not only drug sensitive M.tb , but also clinical MDR and XDR strains, provided proof of concept to repurpose doxycycline to fight MDR-TB and XDR-TB. Further investigations are warranted to clarify the underlying mechanism and optimize the strategy in combination with other anti-TB drugs.

\section{Introduction}

Tuberculosis (TB), a chronic infectious diseases caused by Mycobacterium tuberculosis (M.tb), remains a serious health problem worldwide. Millions of people continue to fall sick with TB each year. In 2018, TB caused about 1.5 million deaths[1]. According to WHO, an estimated 500,000 people worldwide developed MDR-TB in 2017, and an additional 186,772 people contracted rifampicin-resistant TB. It is estimated that about $6.2 \%$ of these cases were XDR-TB[1]. This is the real challenge in the management and treatment of TB, especially MDR-TB and XDR-TB. The treatment of drug susceptible TB requires multiple antibiotics to be taken daily at least 6 months, while the treatment of MDR- and XDR-TB entails second line drugs that are usually toxic for a longer period[2, 3]. Patients often fail to complete their treatment or do not adhere strictly to the regimen, due to the length of the treatment and the toxicity of second line drugs $[2,3]$. Therefore, new drugs with novel mechanisms of action or repurposing the Food and Drug Administration (FDA)-approved drugs are urgently needed to shorten the treatment period and efficiency cure MDR/XDRTB.

Tetracycline antibiotic was first reported in 1948 and found widespread clinical use shortly thereafter [48]. Tetracyclines were known as broad-spectrum antibacterial agents which have a good inhibitory effect 
on gram-positive and gram-negative bacteria, rickettsia, filtered virus, spirochetes and even protozoa [9, 10]. Tetracyclines inhibit bacterial protein synthesis by binding to the $16 \mathrm{~S}$ rRNA of the $30 \mathrm{~S}$ bacterial ribosome subunit, preventing accommodation of incoming aminoacyl-tRNAs at the acceptor site $[11,12]$. Both synthetic and semisynthetic tetracycline analogs have been shown effective against wide ranges of pathogens and different inflammatory diseases and conditions [10], which are the most prescribed oral antibiotics, with a long satisfactory track record of efficacy, low price and safety [13]. Previous studies have showed that doxycycline, a second-generation tetracycline, could efficacy reduce $M$.tb growth both in macrophage and guinea pig TB model via inhibiting host MMPs activity [14]. Doxycycline has been used in vitro against M.tuberculosis[15], and combination with amikacin significantly inhibited 18 of the 29 MDR and XDR TB strains replications [16]. Some of the older members of the tetracycline family also showed activity against $M$. tuberculosis strains in animal models[17].

However, very few studies have been carried out to assess all three generation of tetracyclines activity in mycobacteria. Furthermore, the underlying mechanism of doxycycline direct killing activity is still unclear. Here, we investigated the potential anti-mycobactirum activity of several tetracyclines including demeclocycline, tetracycline, chlortetracycline, tigecycline, oxytetracycline, methacycline, tetracycline and doxycycline in vitro. We further identified two mutants which were resistant to doxycycline by using a transposon M.smeg library.

\section{Materials And Methods}

\section{Bacterial Strains and Culture Conditions}

M.smegmatis mc $^{2} 155$ (M.smeg), M.tb strains H37Ra, H37Rv, and seven clinical isolates: ER17, ER22, $400-13,400-86,400-89,400-123,200-28$ were used in this study. The bacteria were grown in 7H9 Middlebrook broth supplemented with $10 \%$ oleic acid-albumin-dextrose-catalase (OADC, BD), $0.05 \%$ Tween 80 , and $0.2 \%$ glycerol. The culture was incubated at $37^{\circ} \mathrm{C}$, with shaking for 4 to 5 days to achieve midlogarithmic phase $(\mathrm{OD} 600=0.3-0.8)$.

\section{Drugs}

Demeclocycline (hydrochloride), Tetracycline, Chlortetracycline (hydrochloride), Tigecycline, Oxytetracycline, Methacycline (hydrochloride), Tetracycline (hydrochloride) and Doxycycline (hyclate) were purchased from Med Chem Express (MCE, USA). Drugs were diluted and stored according to the manufacturer's instructions.

\section{MIC determination}

The anti-mycobacterium activity was tested using M.smeg, H37Ra, H37Rv and 7 clinical isolates. The bacterial culture was diluted with $7 \mathrm{H} 9-\mathrm{OADC}$ to an $\mathrm{OD}=0.01$ and transferred to a 96 -well microtiter plate 
for the drug sensitivity assay. Serial dilutions of tetracyclines were dispensed into $96-w e l l$ microtiter plates containing bacteria strains. The 96 -well microtiter plates were incubated at $37^{\circ} \mathrm{C}$ with slowly horizontal shaking. The OD600 was measured in a microplate spectrophotometer (BioTek) in indicated times and the growth rate was calculated. The MIC is defined as the lowest concentration of the antimicrobial agent that prevents visible growth of a microorganism under the compound-containing plates [18].

\section{Whole Genome Sequencing of Transposon Mutants}

The transposon M.smeg library was constructed with donor phagemid $\varphi$ MycoMarT7 which includes a transposon that encodes a kanamycin resistance gene[19]. The entire sequence of the $\varphi M y c o M a r T 7$ transposon has been deposited in GenBank (GenBank accession no. AF411123). The transposon library were collected from plates and frozen at $-80^{\circ} \mathrm{C}$. To find strains resistant to doxycycline among the transposon mutants, the transposon M.smeg library were grown to OD600 of 0.8-1.0 in liquid culture. Resistant mutants were isolated by plating $10^{8}$ to $10^{9}$ colony forming units (CFU) of transposon M.smeg library [20]on 7H10-OADC doxycycline plates containing $1 \times$ MICs. Surviving colonies were collected by scraping colonies off plates and restreaked on 7H10-OADC containing Kanamycin and 1×MIC of doxycycline. Genomic DNAs were extracted using the cetyltrimethyl ammonium bromide (CTAB) method[21]. The extracted DNAs from surviving strains were further analyzed by whole genome sequencing (Genewiz, China).

\section{Statistical analysis}

Statistical analysis was performed using GraphPad Prism 7 software. One-way ANOVA was used to assess the effects between more than two groups. Student's t-test was used to assess the effects of only one parameter.

\section{Results}

\section{Tetracycline analogs showed bactericidal activity against mycobacteria in vitro}

8 tetracycline analogs were selected to investigate the antimicrobial effect against Mycobacterium. We first test the effect of these analogs on M.smeg and results showed that seven of the tested tetracycline analogs significantly inhibited M.smeg growth (Fig $1 \mathrm{~A}$ ). Previously studies have showed that tetracycline [22] and tigecycline[23] could significantly inhibited mycobacterial strains growth, which is consistent with our results. We further determined the MIC of four tetracyclines, demeclocycline, chlortetracycline, methacycline and doxycycline. We found that the MICs of M.smeg in $7 \mathrm{H} 9$ broth were $0.067 \mu \mathrm{g} / \mathrm{mL}$, $0.34 \mu \mathrm{g} / \mathrm{mL}, 0.051 \mu \mathrm{g} / \mathrm{mL}$ and $0.064 \mu \mathrm{g} / \mathrm{mL}$ for demeclocycline, chlortetracycline, doxycycline and methacycline (Fig 1 B and Table 2), respectively. The MICs of the M.tb strain H37Ra were $1.25 \mu \mathrm{g} / \mathrm{mL}$, 
$5.15 \mu \mathrm{g} / \mathrm{mL}, 1.03 \mu \mathrm{g} / \mathrm{mL}, 2.39 \mu \mathrm{g} / \mathrm{mL}$ for demeclocycline, chlortetracycline, doxycycline and methacycline (Fig $1 \mathrm{C}$ and Table 2), respectively. We noted that the MICs of those tetracyclines in M.tb strains were about 20 -fold higher than that in M.smeg. The results above demonstrated that some of tetracycline analogs could significantly inhibited mycobacteria growth in vitro.

\section{Activity of Tetracyclines against drug-resistant clinical isolates}

The MICs of demeclocycline, doxycycline and methacycline are lower than that in the peak plasma concentration $[24,25]$. We next assessed the activities of these tetracyclines in clinical isolates.

All the clinical isolates were collected from Shenzhen third people's hospital, China. The isolates including 2 ethambutol resistance strains (ER17, ER22), 1 MDR strain (400-89) and 4 XDR strains (400-13,400$86,400-123,200-28)$. The details of those clinical isolates were showed in Table3.

Three analogs shown anti-Mycobacterium activity for most of the isolates (Table3). The MIC result of three drugs was from 1.28 to $5.13 \mu \mathrm{g} / \mathrm{mL}$ for doxycycline, from 0.25 to $25.06 \mu \mathrm{g} / \mathrm{mL}$ for demeclocycline and 0.59 to $23.9 \mu \mathrm{g} / \mathrm{mL}$ for methacycline (Fig 2 and Table 3). Among these Tetracycline analogs, doxycycline had the lowest MIC value of all the isolates.

\section{Mutation in Porin or MshA showed resistant to doxycycline}

Doxycycline showed better anti-mycobacteria activity among all the tetracyclines used in this study. To study the anti-tuberculosis activity of doxycycline, we used a transposon insertion sequencing-based strategy to identify loci that were required for M.smeg resistance to doxycycline. Mutants were selected for resistance to doxycycline on plates with a concentration of $1 \mathrm{xMIC}$. We isolated 11 resistant clones. Whole genome sequencing identified two genes which exhibited insertions of transposon were candidates for mediating resistance. The less frequently insertion gene was (2 of 11 clones) MSMEG_0933, encoding MshA. Most of the resistant clones (9 of 11) exhibited the insertion of transposon was MSMEG_0965 (Table 4). MshA, a gene encoding the first enzyme involved in the biosynthesis of mycothiol [26], which has been reported to essential for drug susceptibility in M.tb and M.smeg [27-29]. MSMEG_0965 is a porin, which locates in the waxy outer layer of mycobacteria [30] and provides a sieving function. Deletion of porin dramatically increased the resistance of M.smeg to multiple drugs [31-33].

We further tested the MIC of doxycycline with these two mutants. Our results showed both mutants showed increasing resistant to doxycycline and the MICs were $0.51 \mu \mathrm{g} / \mathrm{mL}$ and $0.25 \mu \mathrm{g} / \mathrm{mL}$ for MSMEG_0965 and MSMEG_0933 mutant strains, respectively (Table 5). However, both MshA and porin are non-essential genes [28, 34, 35], it was unlikely to encode the target. The mechanism underlying these two mutants resistant to doxycycline needs to be further investigated.

\section{Discussion}


With rising levels of antibiotic resistance eliminating the drug classes available for treating MDR- and XDR-TB, identifying new drug classes serves a critical need. Additionally, concerning the rising of heritable resistance to PZA [36], novel molecules that are bactericidal against $M$.tb will be important components of much-needed new cocktails for shorter treatment without relapse. In this study, we examined the activity of tetracycline analogs, including three generations of tetracyclines, against mycobacteria. We identified 6 tetracycline analogs (demeclocycline, tetracycline, chlortetracycline, methacycline, tigecycline and doxycycline) that were efficacious against Mycobacteria while oxytetracycline showed poor activity. Our results is consistent with the previously reports on doxycycline and tigecycline $[14,23,37,38]$. We have found that demeclocycline, doxycycline and methacycline were effective against clinical MDR- or XDR-isolates. When comparing the MICs of efficacious tetracyclines, doxycycline had lower MICs among all tetracyclines and is a pre-existing drug approved for the treatment of various infections by gram-positive and gram-negative bacteria, aerobes and anaerobes [39].

Doxycycline is a well-tolerated broad-spectrum oral antibiotic that is used in therapy for many infections.iln this study, 6 of 7 clinical isolates were susceptibility to doxycycline. The result is similar as previously reported doxycycline resistant rate in Russia clinical isolates [15].

Tetracycline-class antibiotics inhibit protein synthesis by preventing attachment of aminoacyl-tRNA to the ribosomal acceptor (A) site that is different with all first line anti-TB drugs [40-42]. Mutations in porin locus show decreased susceptibility to tetracyclines have been described in Gram-negative cells such as E. coli and Helicobacter pylor in previous studies [43-46]. But there is less knowledge of specific genetic requirements for tetracycline inhibitory activity in M.tb. Here, we took advantage of the power of transposon insertion sequencing to identify genetic factors that contribute to the M.tb against doxycycline. In consistent with the previous reports, we found a transposon insertion in MSMEG_0965 which encoding porin[32].Next we examined the role of the porin in the sensitivity of M. smeg to doxycycline by using the mutant of $M$. smeg lacking the porin. The MIC for this mutant increased tenfold. Thus, these results indicate further that doxycycline mainly diffuses through the porin in M. smeg.. In addition, we characterized the molecular basis for doxycycline resistance in mycobacteria, which has not been reported previously. An insertions located in MSMEG_0933, which encodes a glycosyltransferase involved in the first step of mycothiol biosynthesis. Previous study has found that the altered thioldisulfide status can restricts mutant M.smeg ability to grow on plates and at low $\mathrm{pH}$ and to resist challenge by hydrogen peroxide, but it still can grow normally in liquid culture[47]. However, our result found that the MIC for this transposon insertion t increased fivefold, which still showed increasing resistant to doxycycline in liquid culture. To our knowledge, this study is the first to demonstrate that doxycycline resistance in mycobacteria can be associated with the loss of mycothiol function. However, it remains unclear precisely how the altered expression levels of mycothiol drive doxycycline resistance. Elucidating this precise mechanism of action should be the focus of future studies.

\section{Conclusion}


There is a lack of effective drugs to cure MDR/XDR-TB. Our study demonstrated that Tetracycline analogs, especially doxycycline has anti-tuberculosis bactericidal activity. Further studies found a transposon insertion in MSMEG_0933 caused the loss of mycothiol function, which resulted in increased resistant of mutant mycobacteria to doxycycline. Future studies will address whether mycothiol play a role in other tetracycline resistance. If so, the development of agents that inactivate the activity of mycothiol could enable the use of tetracycline antibiotics in M.tuberculosis.. Finally, this study highlights the potential clinical application of tetracycline analogs, especially doxycycline as adjunct antituberculosis drugs.

\section{Abbreviations}

MDR-TB: multidrug-resistant tuberculosis; FDA: Food and Drug Administration (FDA); MIC: minimum inhibitory concentration (MIC); CTAB: cetyltrimethyl ammonium bromide (CTAB); CFU: colony forming units (CFU); XDR-TB: extensively drug-resistant tuberculosis; OADC: acid-albumin-dextrose-catalase; OD: optical density

\section{Declarations}

\section{Acknowledgements}

The authors gratefully acknowledge all the study participants and study staff for the help and cooperation during the study in Shenzhen Third peoples' hospital.

\section{Authors' contributions}

Authors' contributions XC, YC and QO participated in the study design, analysis of data and writing of the manuscript. OQ performed the laboratory examination. DL, GD, and HL collected clinical isolates and organize strains resistant data. All authors read and approved the final version of manuscript.

\section{Funding}

The study was supported by Natural Science Foundation of China (81770013, 81525016 and 81772145$)$, National Science and Technology Major Project (2017ZX10201301), Science and Technology Project of Shenzhen (JCYJ20170412101048337). The funders had no role in study design, data collection and analysis, decision to publish, or preparation of the manuscript.

\section{Availability of data and materials}

All data and materials associated with this study are available from the main text or the additional file. 


\section{Ethics approval and consent to participate}

This study was approved by the Institutional Review Board of the Shenzhen University School of Medicine, China, and informed written consent was obtained from each participant. All experiments and samplings were carried out in accordance with ethical and biosafety protocols approved by the Institutional guidelines.

\section{Consent for publication}

Not applicable

\section{Competing interests}

The authors declare that they have no competing interests

\section{References}

1. Global tuberculosis report 2018: World Health Organization; 2019.

2.Seung KJ, Salmaan K, Rich ML: Multidrug-Resistant Tuberculosis and Extensively Drug-Resistant Tuberculosis. Cold Spring Harbor Perspectives in Medicine 2015, 5(9):a017863.

3.Falzon D,., Jaramillo E,., Schünemann HJ, Arentz M,., Bauer M,., Bayona J,., Blanc L,., Caminero JA, Daley CL, Duncombe C,. WHO guidelines for the programmatic management of drug-resistant tuberculosis: 2011 update. 2011, 38(3):516-528.

4.Duggar BM: Aureomycin: a product of the continuing search for new antibiotics. Annals of the New York Academy of Sciences 1948, 51(2):177-181.

5.FINLAY AC, Hobby G, P’an S, Regna P, Routien J, Seeley D, Shull G, Sobin B, Solomons I, Kane J: Terramycin, a new antibiotic. Science (Washington) 1950:85-87.

6.KING EQ, LEWIS CN, WELCH H, CLARK EA, JOHNSON JB, LYONS JB, SCOTT RB, CORNELY PB: Clinical observations on the use of Terramycin hydrochloride. Journal of the American Medical Association 1950, 143(1):1-4.

7.Roberts MC: Tetracycline resistance determinants: mechanisms of action, regulation of expression, genetic mobility, and distribution. FEMS microbiology reviews 1996, 19(1):1-24.

8.Nelson ML, Levy SB: The history of the tetracyclines. Annals of the New York Academy of Sciences 2011, 1241(1):17-32. 
9.Sum PE, Lee VJ, Testa RT, Hlavka JJ, Ellestad GA, Bloom JD, Gluzman Y,., Tally FP: Glycylcyclines. 1. A new generation of potent antibacterial agents through modification of 9-aminotetracyclines. Journal of Medicinal Chemistry 1994, 37(1):184.

10.Klein NC, Cunha BA: Tetracyclines. Medical Clinics of North America 1995, 79(4):789-801.

11.Brodersen DE, Clemons Jr WM, Carter AP, Morgan-Warren RJ, Wimberly BT, Ramakrishnan V: The structural basis for the action of the antibiotics tetracycline, pactamycin, and hygromycin B on the $30 S$ ribosomal subunit. Cell 2000, 103(7):1143-1154.

12.Wilson DN: The A-Z of bacterial translation inhibitors. Critical reviews in biochemistry and molecular biology 2009, 44(6):393-433.

13.Griffin MO, Fricovsky E, Ceballos G, Villarreal F: Tetracyclines: a pleitropic family of compounds with promising therapeutic properties. Review of the literature. American Journal of Physiology-Cell Physiology 2010, 299(3):C539-C548.

14. Walker NF, Clark SO, Tolu O, Nuria A, Liku T, Shivani S, Luísa S, Bernadette P, Kelly DL, Tree JA: Doxycycline and HIV infection suppress tuberculosis-induced matrix metalloproteinases. American Journal of Respiratory \& Critical Care Medicine 2012, 185(9):989-997.

15.Balabanova Y, Ruddy M, Hubb J, Yates M, Malomanova N, Fedorin I, Drobniewski F: Multidrugresistant tuberculosis in Russia: clinical characteristics, analysis of second-line drug resistance and development of standardized therapy. European Journal of Clinical Microbiology and Infectious Diseases $2005,24(2): 136-139$.

16.Gonzalo X, Casali N, Broda A, Pardieu C, Drobniewski F: Combination of amikacin and doxycycline against multidrug-resistant and extensively drug-resistant tuberculosis. International Journal of Antimicrobial Agents 2015, 45(4):406-412.

17.Hobby GL, Lenert TF: Antituberculous activity of tetracycline and related compounds. American Review of Tuberculosis and Pulmonary Diseases 1955, 72(3):367-372.

18.Irith W, Kai H, Hancock REW: Agar and broth dilution methods to determine the minimal inhibitory concentration (MIC) of antimicrobial substances. Nature Protocols 2008, 3(2):163-175.

19.Sassetti CM, Boyd DH, Rubin EJ: Comprehensive identification of conditionally essential genes in mycobacteria. Proceedings of the National Academy of Sciences 2001, 98(22):12712-12717.

20.Guilhot C, Otal I, Van Rompaey I, Martin C, Gicquel B: Efficient transposition in mycobacteria: construction of Mycobacterium smegmatis insertional mutant libraries. Journal of bacteriology 1994, 176(2):535-539.

21.Parish T, Brown AC: Mycobacteria protocols: Springer; 2008. 
22.Paul C, Muttucumaru DGN, Tanya P: Use of a tetracycline-inducible system for conditional expression in Mycobacterium tuberculosis and Mycobacterium smegmatis. Applied \& Environmental Microbiology 2005, 71(6):3077-3084.

23.Coban AY, Deveci A, Cayci YT, Uzun M, Akgunes A, Durupinar B: In vitro effect of tigecycline against Mycobacterium tuberculosis and a review of the available drugs for tuberculosis. African Journal of Microbiology Research 2011, 5(3):311-315.

24.Agwuh KN, MacGowan A: Pharmacokinetics and pharmacodynamics of the tetracyclines including glycylcyclines. Journal of Antimicrobial Chemotherapy 2006, 58(2):256-265.

25.Newton PN, Chaulet J-F, Brockman A, Chierakul W, Dondorp A, Ruangveerayuth R, Looareesuwan S, Mounier $\mathrm{C}$, White NJ: Pharmacokinetics of oral doxycycline during combination treatment of severe falciparum malaria. Antimicrobial agents and chemotherapy 2005, 49(4):1622-1625.

26.Newton GL, Ta P, Bzymek KP, Fahey RC: Biochemistry of the initial steps of mycothiol biosynthesis. Journal of Biological Chemistry 2006, 281(45):33910-33920.

27.Vilcheze C, Av-Gay Y, Barnes SW, Larsen MH, Walker JR, Glynne RJ, Jacobs WR: Coresistance to Isoniazid and Ethionamide Maps to Mycothiol Biosynthetic Genes in Mycobacterium bovis. Antimicrobial Agents and Chemotherapy 2011, 55(9):4422-4423.

28.Xia X, Catherine V, Yossef AG, Anaximandro GV, Jacobs WR: Precise null deletion mutations of the mycothiol synthesis genes reveal their role in isoniazid and ethionamide resistance in Mycobacterium smegmatis. Antimicrobial Agents \& Chemotherapy 2011, 55(7):3133-3139.

29.Vilchèze C, Av-Gay Y, Attarian R, Liu Z, Hazbón MH, Colangeli R, Chen B, Liu W, Alland D, Sacchettini JC: Mycothiol biosynthesis is essential for ethionamide susceptibility in Mycobacterium tuberculosis. Molecular microbiology 2008, 69(5):1316-1329.

30.Michael F, Michael N, Schulz GE: The structure of a mycobacterial outer-membrane channel. Science 2004, 303(5661):1189-1192.

31.Joachim S, Claudia M, Gilles E, Mamadou D, Michael N: Multidrug resistance of a porin deletion mutant of Mycobacterium smegmatis. Antimicrobial Agents \& Chemotherapy 2004, 48(11):4163.

32.Danilchanka O, Pavlenok M, M: Role of porins for uptake of antibiotics by Mycobacterium smegmatis. Antimicrobial Agents \& Chemotherapy 2008, 52(9):3127.

33.Frenzel E, Schmidt S, Niederweis M, Steinhauer K: Importance of Porins for Biocide Efficacy against Mycobacterium smegmatis. Applied and Environmental Microbiology.

34.Dietmar $\mathrm{H}$, Iris $\mathrm{E}$, Anja T, Michael $\mathrm{N}$ : Expression of the major porin gene mspA is regulated in Mycobacterium smegmatis. Journal of Bacteriology 2007, 189(3):958. 
35.Pavlenok M, Niederweis M: Hetero-oligomeric MspA pores in Mycobacterium smegmatis. Fems Microbiology Letters 2016, 363(7):fnw046.

36.Njire M, Tan Y, Mugweru J, Wang C, Guo J, Yew W, Tan S, Zhang T: Pyrazinamide resistance in Mycobacterium tuberculosis: Review and update. Advances in medical sciences 2016, 61(1):63-71.

37.Rubinstein E, Vaughan D: Tigecycline. Drugs 2005, 65(10):1317-1336.

38. Wallace RJ, Brown-Elliott BA, Crist CJ, Mann L, Wilson RW: Comparison of the in vitro activity of the glycylcycline tigecycline (formerly GAR-936) with those of tetracycline, minocycline, and doxycycline against isolates of nontuberculous mycobacteria. Antimicrobial Agents and Chemotherapy 2002, 46(10):3164-3167.

39.Joshi N, Miller DQ: Doxycycline Revisited. JAMA Internal Medicine 1997, 157(13):1421-1428.

40.Sarkar S, Thach RE: Inhibition of formy/methionyl-transfer RNA binding to ribosomes by tetracycline. Proceedings of the National Academy of Sciences of the United States of America 1968, 60(4):1479.

41.Janin YL: Antituberculosis drugs: ten years of research. Bioorganic \& medicinal chemistry 2007, 15(7):2479-2513.

42.Arbex MA, Varella MdCL, Siqueira HRd, Mello FAFd: Antituberculosis drugs: drug interactions, adverse effects, and use in special situations-part 1: first-line drugs. Jornal Brasileiro de Pneumologia 2010, 36(5):626-640.

43.Mortimer PG, Piddok LJ: The accumulation of five antibacterial agents in porin-deficient mutants of Escherichia coli. Journal of Antimicrobial Chemotherapy 1993, 32(2):195-213.

44.Thanassi DG, Suh G, Nikaido H: Role of outer membrane barrier in efflux-mediated tetracycline resistance of Escherichia coli. Journal of bacteriology 1995, 177(4):998-1007.

45.Pugsley AP, Schnaitman CA: Outer membrane proteins of Escherichia coli VII. Evidence that bacteriophage-directed protein 2 functions as a pore. Journal of bacteriology 1978, 133(3):1181-1189.

46.Trieber CA, Taylor DE: Mutations in the 16S rRNA genes of Helicobacter pylori mediate resistance to tetracycline. Journal of bacteriology 2002, 184(8):2131-2140.

47.Buchmeier NA, Newton GL, Fahey RC: A mycothiol synthase mutant of Mycobacterium tuberculosis has an altered thiol-disulfide content and limited tolerance to stress. Journal of bacteriology 2006, 188(17):6245-6252.

\section{Tables 1, 2, 5}

Table 1 Tetracycline analogs test concentration 


\begin{tabular}{cc}
\hline Drug & $\begin{array}{c}\text { Concentration } \\
(\mu \mathrm{g} / \mathrm{mL})\end{array}$ \\
\hline Deme & 100.3 \\
Tetr & 88.9 \\
Chlo & 103.1 \\
Tige & 117.1 \\
Doxy & 102.7 \\
Oxy & 92.1 \\
Meth & 95.8 \\
Tetr(hy) & 96.2 \\
\hline
\end{tabular}

Deme, Demeclocycline (hydrochloride); Tetr, Tetracycline; Chlo, Chlortetracycline (hydrochloride); Tige, Tigecycline; Doxy, Doxycycline (hyclate) (hyclate); Oxy, Oxytetracycline; Meth, Methacycline (hydrochloride); Tetr(hy), Tetracycline (hydrochloride);

Table 2 MIC of M.smeg and M.tb H37Ra

\begin{tabular}{ccc}
\hline Drug $(\mu \mathrm{g} / \mathrm{mL})$ & M.smeg & M.tb H37Ra \\
& & \\
\hline Deme & 0.067 & 1.25 \\
Chlo & 0.34 & 5.15 \\
Doxy & 0.051 & 1.03 \\
Meth & 0.064 & 2.39 \\
\hline
\end{tabular}

Deme, Demeclocycline (hydrochloride); Chlo, Chlortetracycline (hydrochloride);

Doxy, Doxycycline (hyclate) (hyclate); Meth, Methacycline (hydrochloride); 


\begin{tabular}{cc} 
& $(\mu \mathrm{g} / \mathrm{mL})$ \\
\hline mc $^{2} 155$ mutant MSMEG_0965 & 0.51 \\
$\mathrm{mc}^{2} 155$ mutant MSMEG_0933 & 0.25 \\
$\mathrm{mc}^{2} 155$ wild type $M . s m e g$ & 0.051 \\
\hline
\end{tabular}

Table 5 MIC of Transposon M.smeg

\section{Figures}

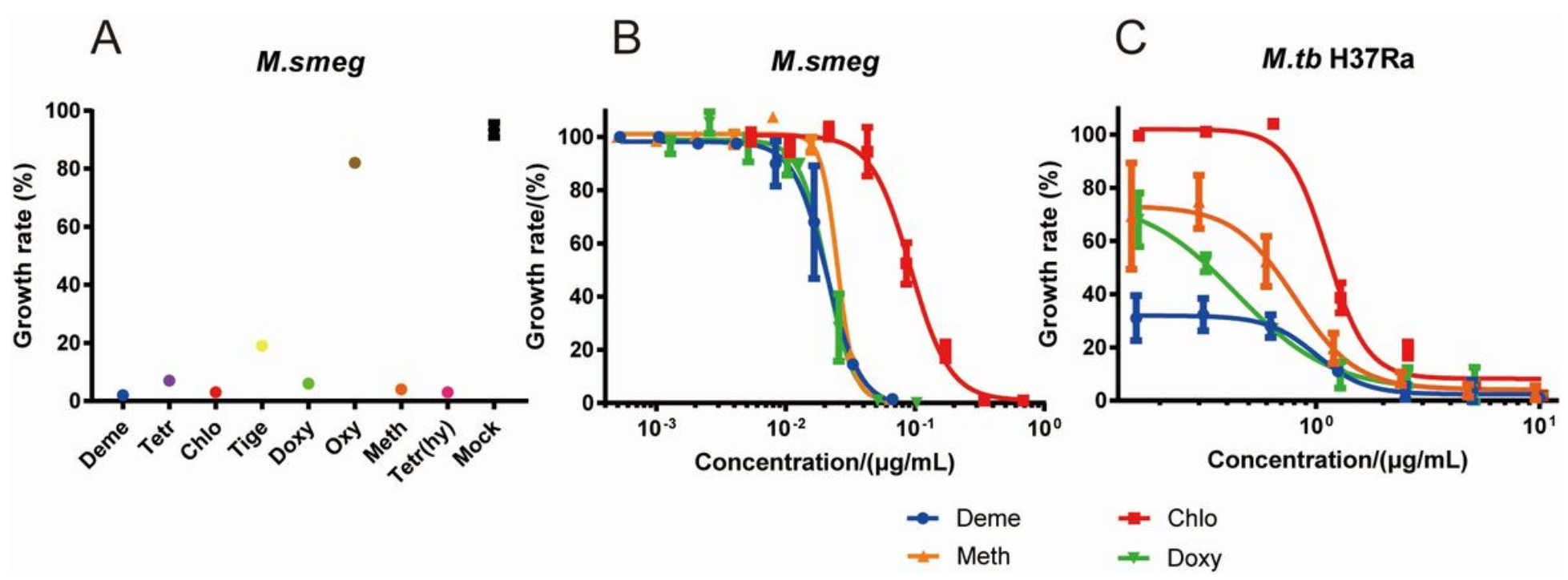

Figure 1

Determination of antimicrobial activity of tetracycline analogs against M.smeg and M.tb H37Ra strains. (A) M.smeg was grown in liquid cultures with eight tetracyclines directly effected on it. Deme, Demeclocycline; Tetr, Tetracycline; Chlo, Chlortetracycline; Tige, Tigecycline; Doxy, Doxycycline; Oxy, Oxytetracycline; Meth, Methacycline; Tetr(hy), Tetracycline (hydrochloride); Mock, none drug. (B) M.smeg was grown below the two fold serially diluted of four tetracyclines; The final test concentration for each tetracyclines ranged from 0.00052 to $0.0667 \mu \mathrm{g} / \mathrm{mL}$ for demeclocycline, 0.0054 to $0.685 \mu \mathrm{g} / \mathrm{mL}$ for chlortetracycline, 0.00129 to $0.103 \mu \mathrm{g} / \mathrm{mL}$ for doxycycline and 0.000498 to $0.0637 \mu \mathrm{g} / \mathrm{mL}$ for methacycline. (C) M.tb H37Ra was grown below the two fold serially diluted of four tetracyclines; The final test concentration for each tetracyclines ranged from 0.16 to $10.02 \mu \mathrm{g} / \mathrm{mL}$ for demeclocycline, 0.16 to $10.31 \mu \mathrm{g} / \mathrm{mL}$ for chlortetracycline, 0.16 to $10.26 \mu \mathrm{g} / \mathrm{mL}$ for doxycycline and 0.15 to $9.58 \mu \mathrm{g} / \mathrm{mL}$ for methacycline. 
A

H37Rv

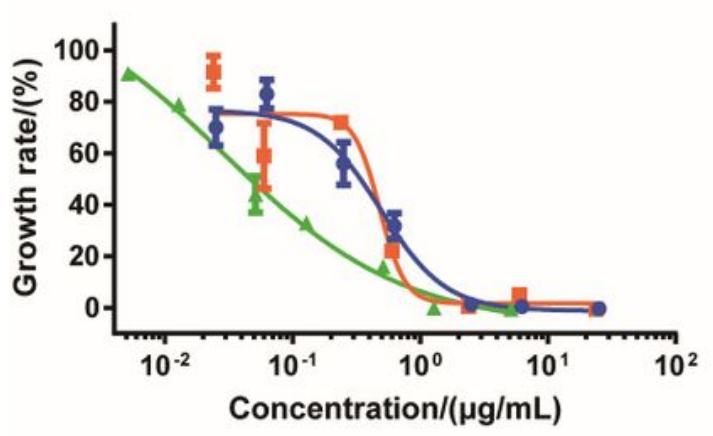

C

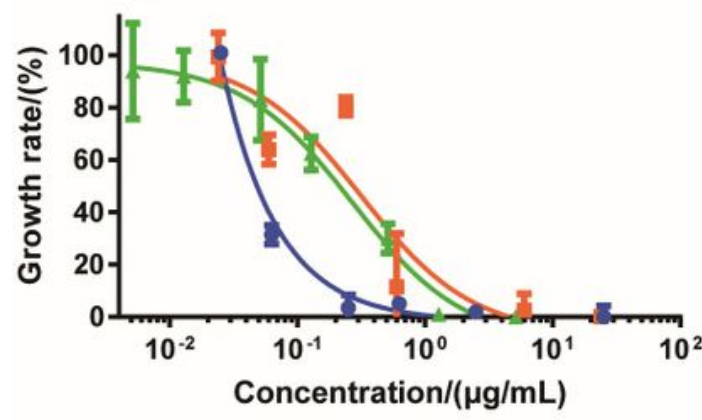

E

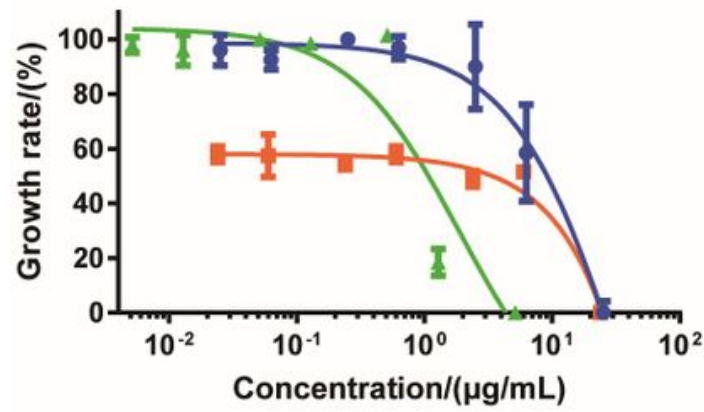

G 400-123

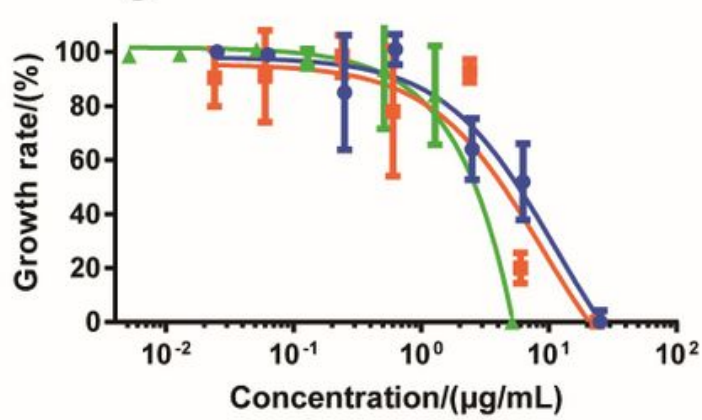

B

ER17

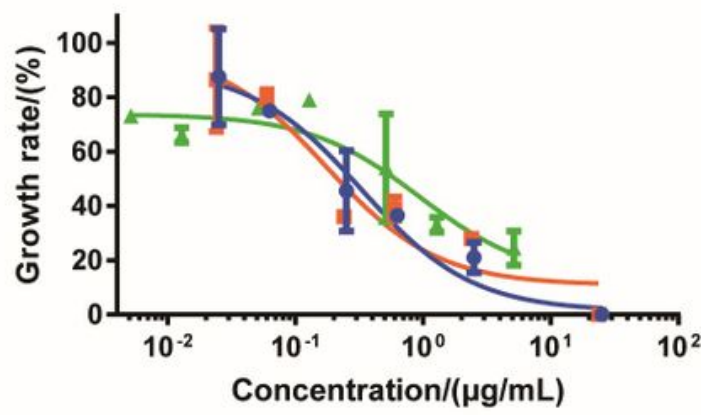

D

400-13

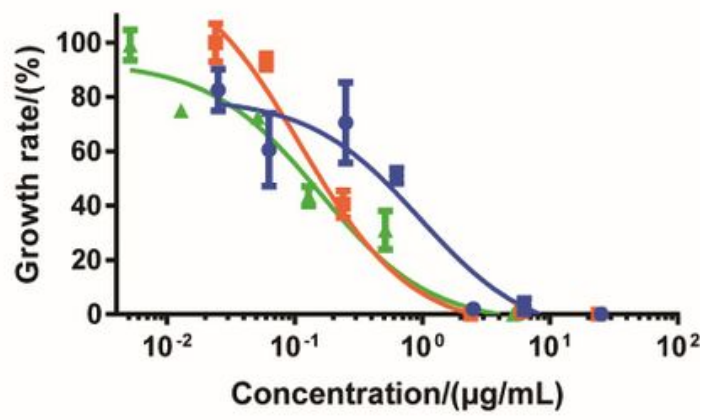

400-89

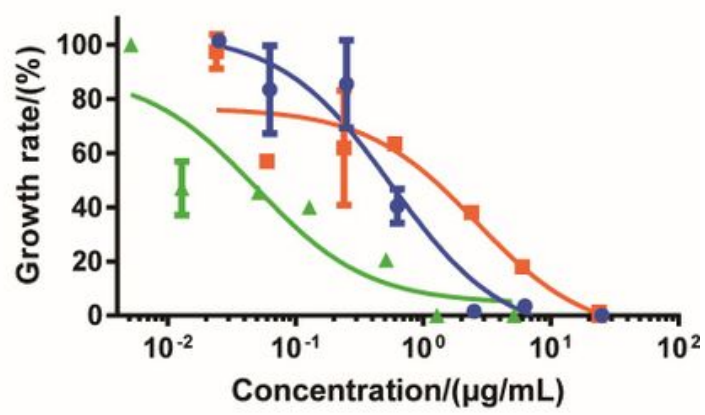

200-28

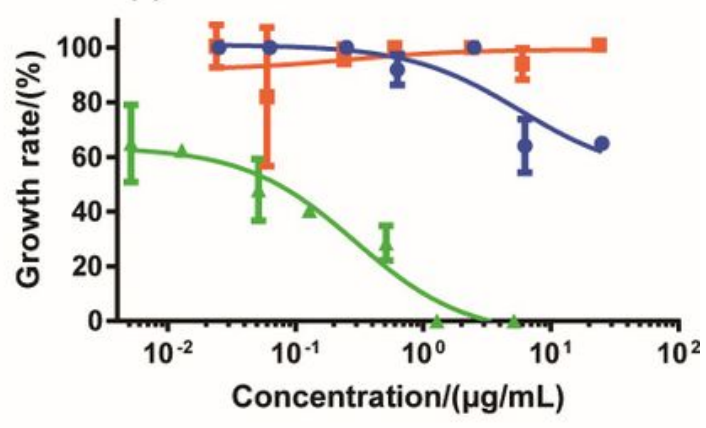

Meth - Doxy

Figure 2

The MIC result of M.tb H37Rv and MDR-TB strain were measured. (A-H) were M.tb H37Rv, Clinical strain ER17, ER22, 400-13, 400-86, 400-89, 400-123, 200-28, respectively. The test concentration range was from 0.00128 to $5.1294 \mu \mathrm{g} / \mathrm{mL}$ for doxycycline, from 0.00627 to $25.066 \mu \mathrm{g} / \mathrm{mL}$ for demeclocycline and 0.00599 to $23.944 \mu \mathrm{g} / \mathrm{mL}$ for methacycline. 


\section{Supplementary Files}

This is a list of supplementary files associated with this preprint. Click to download.

- Table3.xlsx

- Table4.xlsx 